\title{
Nanomaterials for Pharmaceuticals Determination
}

Tawfik A. Saleh*

Department of Chemistry, King Fahd University of Petroleum \& Minerals, Dhahran, Saudi Arabia

*Corresponding author: Saleh TA, Department of Chemistry, King Fahd University of Petroleum \& Minerals, Dhahran 31261 , Saudi Arabia, Tel: +966-13 860 1734; Fax: 966-13 860 1734; E-mail: tawfik@kfupm.edu.sa

Rec Date: May 28, 2016, Acc Date: June 22, 2016, Pub Date: June 25, 2016

Copyright: () 2016 Saleh TA. This is an open-access article distributed under the terms of the Creative Commons Attribution License, which permits unrestricted use, distribution, and reproduction in any medium, provided the original author and source are credited.

\begin{abstract}
Nanomaterials play an important role in the fabrication of many devices and modified materials, due to their unique properties, such as a large surface area/volume ratio and their high mechanical strength. The applicability of nanomaterials in drug determinations is being well investigated. This review provides a summary of the synthesis methods, characterization routes, and applications toward drugs. Nanomaterials have been investigated for their applications with electrochemical and spectroscopic systems for drug determinations. Most of the applications described in the literature are concerned with pushing the limits of how few molecules can be detected in a given volume of solution. Nanomaterials based methods are useful because of their excellent electrical, thermal, magnetic, and optical properties. The real value of the designed method lies in their detection limit range, sensitivity, and other merits that can be enhanced by the nanomaterials modified electrodes or surfaces.
\end{abstract}

Keywords: Nanomaterials, Synthesis, applications, drugs

\section{Introduction}

A nanomaterial is a broad term given to the materials found at the nanoscale. It is a material that has a unit size between the ranges of $1-100 \mathrm{~nm}$. Nanomaterials can be naturally occurring or chemically, mechanically, physically, or biologically synthesized with various structures, Figure 1. The various structures of carbon-based nanomaterials are depicted in Figure 2.

A simple classification of nanomaterials is based on their structures includes zero dimension, one dimension, two dimensions, and three dimensions. Nanoparticles have unique chemical and physical properties as compared to their solid bulk materials because of their high surface area, and electronic properties in addition to the controlled morphology.

Figure 3 presents examples of various morphological structures of nanomaterials. These particles have been utilized in many applications, for example, electrochemistry, photochemical, and biomedicine [1-3]. Nanoparticles have many functional platforms, and they can be utilized for imaging and therapeutic functions. These platforms can be prepared from various inorganic and organic materials, but the inorganic platforms are very important for simultaneous therapy and diagnosis because of their easy modification, high drug loading capacity, and stability [4].

In addition, nanoparticles can be used for targeted drug delivery and the metallic nanoparticles respond resonantly to the magnetic field because they have very small sized particles, to transfer in the tumor cells as hyperthermia agents [5]. Nanomaterials can be utilized for the determination of the drugs [6-9].

\section{Synthesis of the Nanomaterials}

There are two different approaches to preparing nanomaterials- the bottom-up approach and the top-down approach. In the bottom-up approach, the nanoparticles are first obtained at the atomic level and later integrated into the desired material. The top-down approach starts with a bulk material at the macroscopic level followed by trimming of the material to the desired nanoparticles.

Many methods can be used for the synthesis of nanoparticles including chemical, physical, photochemical and biological procedures [10-17]. The type of method and the experimental conditions determine the shape and the size of the nanoparticles. The selection of any of these methods in terms of costs, scalability, particle sizes and size distribution should be considered. Generally, chemical methods provide an easy way to synthesize nanoparticles in solution.

The most widely used chemical methods for the synthesis of nanoparticles are (i) solid-state reaction; (ii) sol-gel technique; (iii) solvothermal method; (iv) hydrothermal method; and (v) molten salt method.

The most widely used physical methods for the synthesis of nanoparticles are dry and wet mechanical grindings which are methods of choice for inexpensive nanoparticle preparation. For technological applications, wet grinding is preferable because it allows more options to control the size of the nanoparticles.

In the physical methods generally, it is required to have; (i) raw material to grind; (ii) surfactant to cover the particle surface and prevent their aggregation and overheating during grinding, example is oleic acid; and (iii) fluid carrier where both raw material and surfactant are mixed with fluid carrier; example is heptane.

Physical-chemical methods are also used to prepare nanoparticles with controllable sizes and shapes a combination of chemical methods with external physical factors (for example, a chemical reaction in the presence of electromagnetic fields or mechanical milling) is needed.

Biological methods are used to prepare some kinds of nanomaterials. It allows the production of very small nanoparticles 
Page 2 of 6

(5-10 nm). Biological methods are proposed as eco-friendly "green" alternatives to existing chemical and physical methods. The biosynthesis of different types of nanoparticles are available $[18,19]$. It should be mentioned that during the preparation of the nanomaterials, safety precautions should be considered as the nanoparticles may penetrate and cause some effects $[20,21]$.

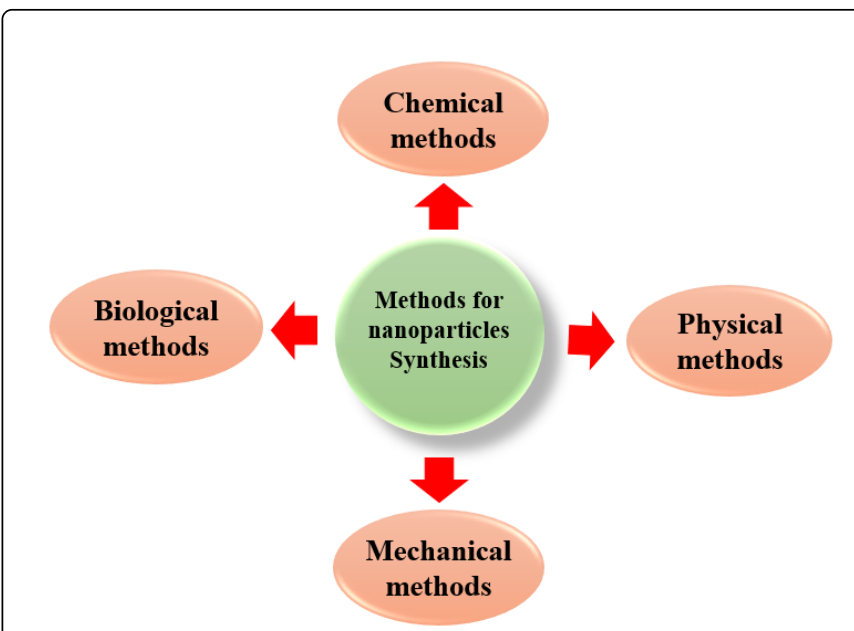

Figure 1: Different methods for the synthesis of nanoparticles.
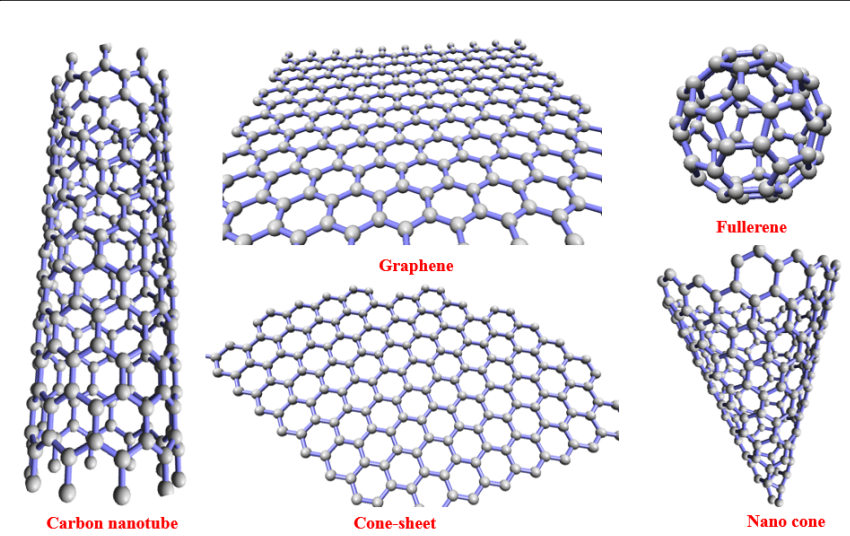

Figure 2: Various structures of carbon-based nanomaterials.

\section{Characterization of the Nanomaterials}

Various techniques are being utilized for the characterization of nanomaterials including X Ray Diffraction (XRD), field emission scanning electron microscope (FESEM), high resolution transmission electron microscope (HRTEM), Energy-dispersive X-ray spectroscopy (EDX), X-ray photoelectron spectroscope (XPS).and infrared transform absorption spectrometer (FTIR); to explain the morphology of the composites, crystalline phases and average size [22, 23].

The morphology of the composites can be characterized by SEM, AFM and TEM. Examples of different morphologies are depicted in Figure 3. The presence of functional groups on the surface of nanotubes can be carried out by FTIR, Raman, XPS and EDX. The thermal stability cab be studied by thermogravimetric (TGA). The surface area, porosity, pore size and pore distributions can be evaluated by physisorption and chemisorption analyzers. Temperature programmed desorption (TPD) analyses determine the number, type and strength of active sites available on the surface of a nanomaterial by measuring the amount of gas desorbed at various temperatures.

If multiple active materials are present, the gas desorbs at different temperatures. Temperature programmed reduction (TPR) determines the number of reducible metal species present in the nanomaterial and the temperature at which reduction occurs. This can be performed by measuring the amount of analysis gas (like hydrogen) that reacts with the catalyst at different temperatures. Temperature programmed oxidation (TPO) provides the extent to which the nanomaterials can reoxidize.

Nanoparticles are being developed for a range of healthcare product applications including drug delivery, controlled release systems, and medical devices. These products present multiple challenges for product characterization that require sophisticated analytical techniques operated by experienced analysts, Figure 4 .

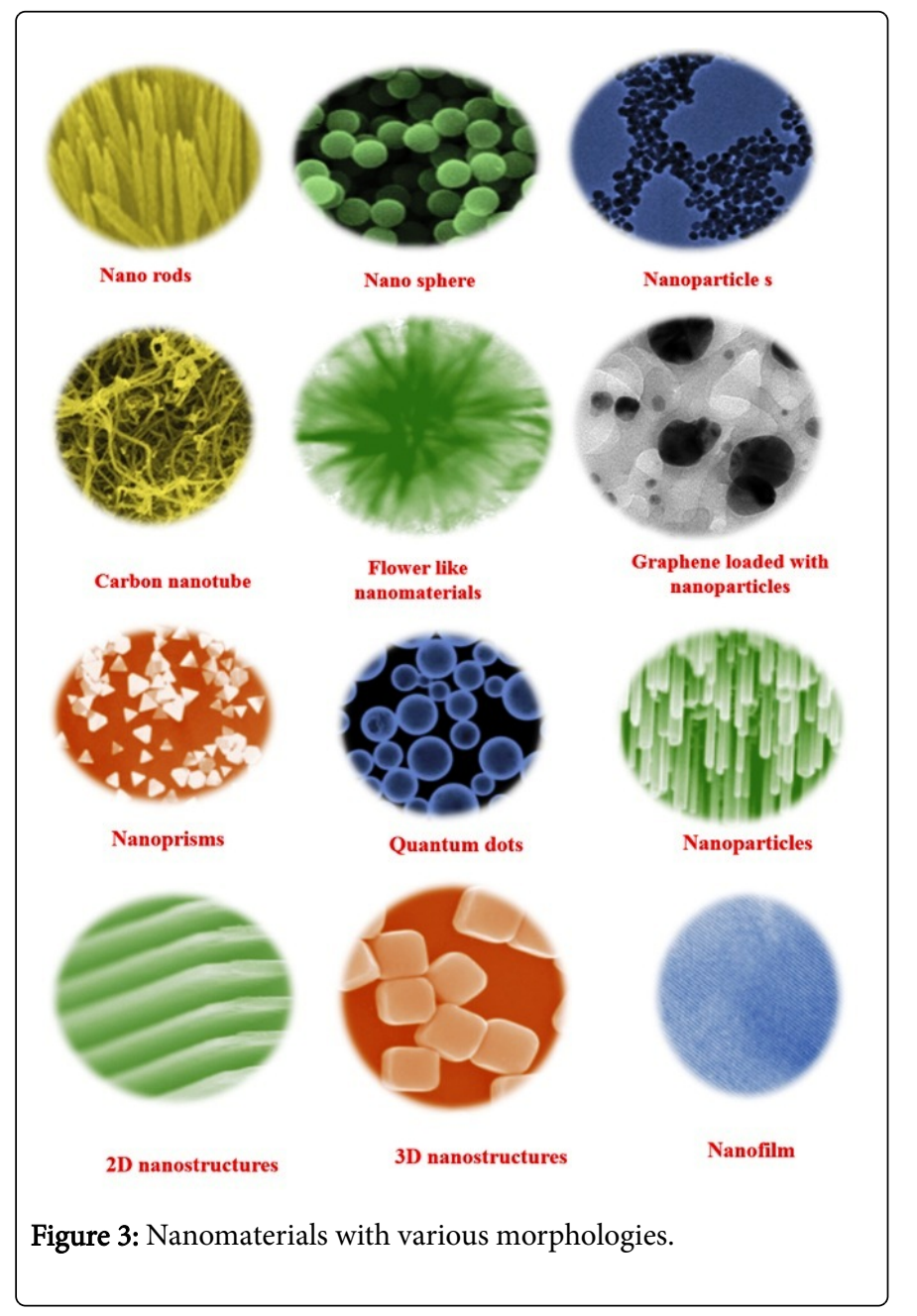




\section{Characterization of nanoparticles}

SEM and TEM: Analysis of particle size and morphology using electron microscopy

$>$ Particle size determination using light scattering techniques or disc centrifugation

$>$ Zeta potential measurements for nanoparticle interactions to aid formulation development

$>$ Physical chemical properties such as viscosity, solubility

BET isotherm: Surface area and porosity measurements

$>$ Crystal morphology by powder X-Ray Diffraction

Controlled release laboratory investigations

Figure 4: Various techniques for the nanomaterials characterization

\section{Application of the Nanomaterials in Drugs Analysis}

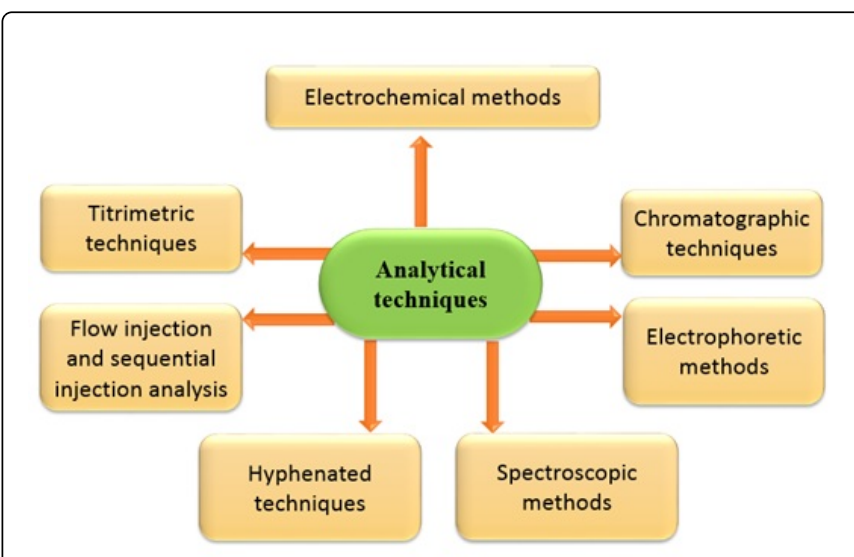

Figure 5: Classification of general analytical techniques for drugs determination

The discovery of nanomaterials such as carbon-based nanomaterials, including carbon nanotubes, and graphene, have garnered tremendous interest for their potential in the design of high performance spectroscopic and electrochemical sensor platforms due to their exceptional thermal, mechanical, electronic, and catalytic properties. There are various analytical techniques for drugs determination including (i) titrimetric techniques, (ii) chromatographic techniques such as thin layer chromatography, highperformance liquid chromatography and gas chromatography; (iii) spectroscopic techniques such as spectrophotometry, near infrared spectroscopy, nuclear magnetic resonance spectroscopy, fluorimetry and phosphorimetry; (iv) electrochemical methods; (v) electrophoretic methods; and (vi) flow injection and sequential injection analysis. Figure 5 depicts classification of general analytical techniques for drugs determination.

\section{Electrochemical sensors and biosensors}

Several methods have been developed for the determination of drugs such as chromatographic techniques, spectrophotometry and capillary zone electrophoresis. However, most of these methods need expensive and sophisticated instruments and are time consuming, and in many cases, due to their relatively low sensitivities, they need a preliminary pre-treatment step, which is laborious and expensive.

Electroanalytical techniques are excellent and well known methods for ketoconazole determination in pharmaceutical formulations, cosmetics and in biological fluids by lower cost, shorter analysis time and no need of sample pretreatment compared to chromatographic techniques. The ketoconazole electrochemical properties were studied with the application of polarographic and Voltammetric techniques. The electrochemical methods described in the literature for determination of drugs such as ketoconazole were the use of mercury electrode, silver solid amalgam electrode carbon paste electrode, platinum, gold and glassy carbon electrode [24-29]. The sensitivity of 
Page 4 of 6

the said methods permits them to be applied for determining ketoconazole in blood, urine, pharmaceutical preparations, and cosmetics. In the electroanalytical determination of pharmaceutical products, the foremost drawback is related to electrode surface fouling due to adsorption of the reactants from the solution interface or the reaction products of the electrochemical reactions, which leads to low sensitivity and reproducibility. In recent years, the increasing use of carbon paste electrodes (CPEs) modified by nanomaterials for electroanalytical measurement of a variety of organic species of biological and pharmaceutical importance has been reported [30-33].

The materials and biomaterials with rich nanostructures not only improve the electronic properties and increase the effective electrode surface for transferring electrochemical signal but also produce detectable signals for indirect detection of targets. Nanotechnology has advanced the progress of electrochemical sensors and biosensors. Nanomaterial-modified detection systems represent a chief driver towards the adoption of electrochemical methods, since nanomaterials enable functional tunability, ability to self-assemble, and novel electrical, optical and catalytic properties that emerge at this scale. This results in tremendous gains in terms of sensitivity, selectivity, and versatility because the nano-sized modifiers influence the electron transfer. Example is the use of nanowires for the development of efficient electrochemical sensors and biosensors due to their advantages such as mechanical stability, lightweight, enhancement in the current, reduction in potential. Functional nanowire arrays constitute one of the most fascinating nanotechnology [34]. Compared to other methods, such as spectroscopy and chromatography, the electrochemical measurements are relatively cheaper, simpler, and easier to miniaturize, which makes them more suitable for portablebased detection. Various strategies are used to improve the efficacy of sensing. Signal amplification for detection to utilize nanomaterials as carriers or tracers, catalysts, and electronic conductors and produce a synergic effect among catalytic activity, conductivity, and biocompatibility has been achieved.

\section{Application of Surface Raman enhanced Scattering (SERS) in drugs analysis}

The Surface Raman enhanced Scattering (SERS) have attracted considerable attention for the sensitive detection of a variety of biological and pharmaceutical compounds. It is one of the most important methods for the detection and characterization of drugs in pharmaceutical samples. SERS is a very promising method for investigating the pharmaceutical compounds, due to its an ability to provide information about chemical structural and physical properties and identifies substances from the characteristic spectra [35]. The major advantage of SERS over other spectroscopic techniques is that SERS is a molecularly specific technique producing spectra, which has sharp peaks, whereas, for example, fluorescence spectra is broad, overlapping, and less specific for a particular molecule [36]. The average enhancement factor of Raman signals by SERS has been recorded in excess of 1010 for the detection of the analyte on the surface of nanomaterials, which is related to the excitation of surface plasmon resonance on substrates as a result of the interactions between adsorbents and metals [37].

SERS involves adsorption of molecules onto the substrate surface. SERS enhancement has an influence on the type of metallic substrates. Most common metals used are gold, silver, and copper nanoparticles. Among them, the silver metal is the most common type used as SERS substrates, due to its stability in the air, and very strong surface
Plasmon resonance in UV-vis, and near IR range. The silver nanoscale $\mathrm{nm}$ display localized surface Plasmon's resonance that can be excited with visible light, causing a high enhancement compared with all the metals. However, silver nanoparticles have a strong tendency to aggregate over storage time, which leads to low detection reproducibility and reliability.

The enhancement can be explained by two mechanisms; chemical enhancement, and electromagnetic enhancement. The chemical effects are contributing the minor part of the enhancement magnitude, while the electromagnetic effect is dominant of the SERS signal. The electromagnetic enhancement is dependent on the roughness that present metal surface, which is related to the surface Plasmon resonance of the metal surface. The electromagnetic enhancement involves excites surface Plasmon on the metal, due to the incident laser irradiates on the metal surface. This excitation surface Plasmon leads to generate a strong electromagnetic field on the metal surface that magnifies the incident laser and the Raman scattering. The electromagnetic enhancement is generally of the order of 106-108 . While the chemical enhancement is dependent on charge transfer excitation between the adsorbent and metal surfaces through their bonds, which can occur by transfer one electron from the highest occupied to the Fermi level of the metal, or by transfer one electron from the Fermi level of the metal to the lowest unoccupied molecular orbital. These charge transfers cause a change in the polarizability of the molecule. The chemical enhancement can be normally achieved up to $102-103$.

On the other side, electrochemical methods have many advantages like easy handling, short analysis time, very sensitive, no use of organic solvent, the ability to detect multiple analytes without separation steps, the ability to analyze targets within biological matrices, including sweat or urine when compared with other techniques. Electrochemical methods have been shown to be excellent in the pharmaceutical formulation, and for the determination of the drug in bulk structure. Therefore, many of the studies have been reported to pharmaceutical analysis, including the voltammetric determination of antiamoebic, capecitabine, sedatives, antiadrenergic, ketoconazole, clotrimazole, and piperazine. Moreover, the electrochemical methods are very helpful techniques to study the reaction mechanism. Redox properties of a pharmaceutical compound can give insights into its metabolic fate or pharmaceutical activity. The important idea in electrochemical studies is based on modifying working electrodes by nanomaterials like silver, and gold nanoparticles, which provide high surface area, electronic and mechanical properties, increase the rate of electron transfer.

\section{Hyphenated techniques}

The coupling of a separation technique and on-line separation technique leads to the development of a hyphenated technique. The last two decades saw a remarkable advancement in the hyphenated techniques and its application in pharmaceutical analysis. A variety of hyphenated techniques such as liquid chromatography combined with mass spectrometry, gas chromatography combined with mass spectrometry, liquid chromatography - nuclear magnetic resonance spectroscopy, capillary electrophoresis inductively coupled plasmamass spectroscopy and capillary electrophoresis with mass spectrometry have been applied in the analysis of pharmaceuticals. The determination of drugs in biological materials is an important step in drug discovery and drug development. The determination of drugs in biological materials is an important step in drug discovery and drug development. HPLC together with various types of detection such as 
Page 5 of 6

ultraviolet, fluorescence, and mass spectrometry has become the method of choice for bioanalytical method development [38]. HPLC with UV or MS/MS' detection is presented for the analysis of meloxicam in biological samples and pharmaceutical formulations [39]. Liquid chromatography-electrospray ionization-mass spectrometry method for the qualitative and quantitative determination of metabolites after oral administration of Rhizome coptidis and Zuojinwan preparation in rat urine has been developed. Other techniques include matrix-assisted laser-desorption ionization time-of-flight mass spectrometry (MALDI-TOF MS), and fluorescence. One of these methods is matrix-assisted laser-desorption ionization time-of-flight mass spectrometry (MALDI-TOF MS), which is a powerful technique for DNA analysis. MALDI-TOF MS approaches have been developed for rapid screening of single nucleotide polymorphisms (SNPs), epigenotype analysis, quantitative allele studies, and for the discovery of new genetic polymorphisms. These methods are based on single base primer extension and minisequencing implemented with new chemicals using MALDI-TOF MS and include photochemical and other chemical and enzyme cleavage strategies that facilitate sample automation and MS analysis for real-time genotyping and resequencing screening [40-43].

\section{Acknowledgment}

The authors would like to acknowledge the support provided by King Abdulaziz City for Science and Technology (KACST) through project No. A.T.34-8. The authors would like also to acknowledge the support by King Fahd University of Petroleum and Minerals (KFUPM).

\section{References}

1. Haruna K, Saleh TA, Thagfi AJ, Saadi AAA (2016) Structural properties, vibrational spectra and surface-enhanced Raman scattering of 2, 4, 6 trichloro-and tribromoanilines: A comparative study? Journal of Molecular Structure 1121: 7-15.

2. TA Saleh (2014) Detection: from electrochemistry to spectroscopy with chromatographic techniques, recent trends with nanotechnology? Detection 2: 27-32.

3. Kim D, Jon S (2012) Gold nanoparticles in image-guided cancer therapy. Inorgan Chim Acta 393: 154-164.

4. Khlebtsov NG, Dykman LA (2010) Optical properties and biomedical applications of plasmonic nanoparticles. J Quantitat Spectroscop Radiat Transf 111: 1-35.

5. Tedesco S, Doyle H, Blasco J, Redmond G, Sheehan D (2010) Oxidative stress and toxicity of gold nanoparticles in Mytilus edulis. Aquat Toxicol 100: 178-186.

6. TA Saleh (2014) Spectroscopy: between modeling, simulation, and practical investigation? Spectral Analysis Review 2: 1-2.

7. TA Saleh, VK Gupta (2014) Covalent and non-covalent functionalization of carbon nanotubes? Advanced Carbon Materials and Technology. pp: 317-330.

8. TA Saleh (2011) Sensing of chlorpheniramine in pharmaceutical applications by sequential injector coupled with potentiometer? Journal of Pharmaceutical Analysis 1: 246-250.

9. AM Idris, AEE Ibrahim, AM Abulkibash, TA Saleh, KEE Ibrahim (2011) Rapid inexpensive assay method for verapamil by spectrophotometric sequential injection analysis? Drug testing and analysis 3: 380-386.

10. Wei, X, Xu G, Ren Z, Wang Y, Shen G, et al. (2008) Synthesis of Highly Dispersed Barium Titanate Nanoparticles by a Nov- el Solvothermal Method. J Am Ceram Soc 91, 315-318.

11. Hayashi H, Hakuta Y (2010) Hydrothermal Synthesis of Metal Oxide Nanoparticles in Supercritical Water. Materials3, 3794-3817.
12. Zhang, H. et al. (2011) Fabrication of Monodispersed 5-nm BaTiO3 Nanocrystals with Nar- row Size Distribution via One-Step Solvothermal Route. J Am Ceram Soc 94: 3220-3222.

13. Atkuri H, Cook G, Evans DR, West J, Zhang K, et al. (2009) Preparation of ferroelectric nanoparticles for their use in liquid crys- talline colloids. Journal of Optics A: Pure and Applied Optics 11: 024006.

14. Garbovskiy Y, Glushchenko A (2010) in Solid State Physics (ed. Camley, R.E.) $1-74$

15. Cook G1, Glushchenko AV, Reshetnyak V, Griffith AT, Saleh MA, et al. (2008) Nanoparticle doped organic-inorganic hybrid photorefractives. Opt Express 16: 4015-4022.

16. Li X, Xu H, Chen ZS, Chen G (2011) Biosynthesis of Nanoparticles by Microorgan- isms and Their Applications. J Nanomater 2011: 270974.

17. Chen CL1, Rosi NL (2010) Peptide-based methods for the preparation of nanostructured inorganic materials. Angew Chem Int Ed Engl 49: 1924-1942.

18. Briggs BD, Knecht MR (2012) Nanotechnology Meets Biology: Peptidebased Methods for the Fabrication of Functional Materials. J Phys Chem Lett 3: 405-418.

19. Dhillon GS1, Brar SK, Kaur S, Verma M (2012) Green approach for nanoparticle biosynthesis by fungi: current trends and applications. Crit Rev Biotechnol 32: 49-73.

20. Saleh TA (2011) Testing the effectiveness of visual aids in chemical safety training, Chem Health Saf 18: 3-8.

21. Saleh TA (2013), A strategy for integrating basic concepts of nanotechnology to enhance undergraduate nano-education: Statistical evaluation of an application study?, J Nano Educ 4: 1-7.

22. Saleh TA (2011) The influence of treatment temperature on the acidity of MWCNT oxidized by HNO 3 or a mixture of HNO3/H2SO4, Appl Surf sci 257: 7746-7751.

23. Saleh TA (2013) The role of carbon nanotubes in enhancement of photocatalysis?; Syntheses And Applications Of Carbon Nanotubes And Their Composites, 479-493.

24. Dantas ANDS, Souza DD, Lima JESD, Lima-Neto PD, Correia AN, et al. (2010) Voltammetric determination of ketoconazole using a polished silver solid amalgam electrode, Electrochimica Acta 55: 9083-9089.

25. Shamsipur M, Farhadi K (2000) Adsorptive stripping voltammetric determination of ketoconazole in pharmaceutical preparations and urine using carbon paste electrodes, Analyst, 125: 1639-1643.

26. Arranz P, Arranz A, Moreda JM, Cid A, Arranz JF (2003) Stripping voltammetric and polarographic techniques for the determination of anti-fungal ketoconazole on the mercury electrode. J Pharm Biomed Anal 33: 589-596.

27. Kumar KG, Augustine P, John S, Radecki J, Radecka H, (2008) Fabrication of potentiometric sensors for the selective determination of ketoconazole, Analytical Letters, 41: 1144-1157.

28. Ries ME, Ghany MA, Hussin L, El-Anwar FM, Mohamed A, (2013) Voltammetric behavior of ketoconazole and its determination in cosmetic preparation using a $B$-cyclodextrin modified glassy carbon electrode, Bulletin of Faculty of Pharmacy, Cairo University, 51: 49-55.

29. Saleh TA, Abulkibash AM, Ibrahim AE (2012) Portable system of programmable syringe pump with potentiometer for determination of promethazine in pharmaceutical applications?, Saudi Pharmaceutical Journal 20: 155-160.

30. Posac JR, Vázquez MD, Tascón ML, Acuña JA, Fuente CDL, et al. (1995) Determination of aceclofenac using adsorptive stripping voltammetric techniques on conventional and surfactant chemically modified carbon paste electrodes, Talanta, 42: 293-304.

31. Fernandez-Abedul M, Velazquez-Rodriguez M, Barreira-Rodriguez J, Costa-Garcia A, (1997) Voltammetric Determination of Naltrexone in Pharmaceuticals, Analytical letters, 30: 1491-1502.

32. Khodari M, Mansour H, Salah El-Din H, (1997) Preconcentration and determination of the tricyclic antidepressant drug-imipramine at modified carbon paste electrode, Analytical letters, 30: 1909-1921. 
33. Lukasiewicz KM, Roginska K (2014) Voltammetric determination of antifungal agents in pharmaceuticals and cosmetics using boron-doped diamond electrodes, Analytical Methods, 6: 7912-7922.

34. Yogeswaran U, Chen SM (2008) A Review on the Electrochemical Sensors and Biosensors Composed of Nanowires as Sensing Material Sensors 8: 290-313.

35. Le Ru EC, Blackie E, Meyer M (2007) Surface Enhanced Raman Scattering Enhancement Factors:? A Comprehensive Study. J. Phys. Chem. C. 111: 1379- 13803.

36. Gupta R, Weimer WA (2003) High enhancement factor gold films for surface enhanced Raman spectroscopy. Chemical Physics Letters. 374: 302-306.

37. Xua S, Jiang S, Wang J, Wei J, Yue W, et al.(2016) Graphene isolated Au nanoparticle arrays with high reproducibility for high-performance surface-enhanced Raman scattering. Sensors and Actuators B. 222 1175-1183.

38. Siddiqui MR, AlOthman ZA, Rahman N (2013) Analytical techniques in pharmaceutical analysis: A review, Arabian Journal of Chemistry.

39. Brezovska M, Jampilek J, Opatrilova R (2001) A Review of HPLC Methods Used for Determining the Presence of Meloxicam Curr. Pharm. Anal 9: 69-76.
40. Abdelhamid HN, Hui-Fen Wu, (2015) Synthesis and characterization of quantum dots for application in laser soft desorption/ionization mass spectrometry to detect labile metal-drug interactions and their antibacterial activity. RSC advances 5: 76107-76115.

41. Abdelhamid HN1, Wu HF (2013) Furoic and mefenamic acids as new matrices for matrix assisted laser desorption/ionization-(MALDI)-mass spectrometry. Talanta 115: 442-450.

42. Abdelhamid HN, Wu HF, (2012) A method to detect metal-drug complexes and their interactions with pathogenic bacteria via graphene nanosheet assist laser desorption/ionization mass spectrometry and biosensors, Anal Chim Acta 751: 94- 104.

43. Nadeem Khan, Hani Nasser Abdelhamid, Jhen-Chen Wang, Ja-Yi Yan, Fon-Tsai Chung, Hui-Fen Wu, High order tandem mass spectrometry (MS4) for flutamide structural analysis from pharmaceutical formulations in Electrospray ion trap mass spectrometry, Analytical Chemistry Research, 2015, 3, 89-97. 